\title{
Rosai-Dorfman disease of the lung with features of obliterative arteritis
}

\author{
Jose G. Mantilla ${ }^{1}$ - Anna Shmukler ${ }^{2}$ - Yanhua Wang ${ }^{3,4}$
}

Received: 26 January 2016 / Accepted: 1 June 2016 / Published online: 7 June 2016

(C) Springer-Verlag Berlin Heidelberg 2016

\begin{abstract}
Sinus histiocytosis with massive lymphadenopathy, also known as Rosai-Dorfman disease (RDD), is a rare benign non-Langerhans cell histiocytosis. Twenty-five to forty percent of the cases are extranodal and have been reported in virtually all anatomic locations. In this article, we report a highly unusual case of RDD which presented as multiple pulmonary nodules and associated hilar lymphadenopathy. On resection, extensive obliterative arteritis was noticed. This pathologic presentation of RDD has not been reported before in the English literature. Accurate recognition of this entity is crucial to prevent unnecessary aggressive treatment.
\end{abstract}

Keywords Rosai-Dorfman disease $\cdot$ Lung $\cdot$ Obliterative arteritis

\section{Introduction}

Sinus histiocytosis with massive lymphadenopathy (also known as Rosai-Dorfman Disease) is a rare, benign disorder,

Yanhua Wang

ywang@montefiore.org

1 Department of Pathology, Albert Einstein College of Medicine -Montefiore Medical Center, 111E 210th Street, Bronx, NY 10467, USA

2 Department of Radiology, Albert Einstein College of Medicine -Montefiore Medical Center, 111E 210th Street, Bronx, NY 10467, USA

3 Department of Pathology - Division of Hematopathology, Albert Einstein College of Medicine - Montefiore Medical Center, 111E 210th Street, Bronx, NY 10467, USA

4 Silver Zone, Floor 4, Surgical Pathology, Bronx, NY 10467, USA described by Rosai and Dorfman in 1969 [1, 2], and is characterized by painless lymphadenopathy in children and young adults with no sex preference [1-4]. Up to $40 \%$ of the cases of Rosai-Dorfman disease (RDD) are extranodal and have been reported in virtually every organ in the body [3].

The clinical presentation of RDD is variable, making it challenging to diagnose; low grade fever and weight loss are occasional manifestations. RDD disease is a self-limited condition, which completely and spontaneously improves in 20 $50 \%$ of patients, with a worse overall prognosis seen in extranodal forms, particularly those involving the liver, kidneys, and lower respiratory tract [2]. Early diagnosis of this condition can prevent unnecessary medical intervention.

Histopathological examination via surgical biopsy is essential for its diagnosis. The characteristic histologic findings of RDD are marked expansion of the subcapsular sinuses with abundant plasma cells and histiocytes, with the latter classically showing multiple viable lymphocytes in their cytoplasm (emperipolesis) [1-4]. Extranodal forms of the disease are often difficult to diagnose and can be recognized by the presence of histiocytes with emperipolesis, commonly in a background of expansile collagen fibrosis.

In this article, we report an unusual case of combined nodal and extranodal RDD in the lung, which presented as a central pulmonary mass with associated hilar lymphadenopathy, and histologically showed extensive obliteration of medium to large arterial vessels.

Immunohistochemical staining reveals the characteristic phenotype: S100-positive, CD68-positive, and CD1a-negative. Immunoreactivity to EBV and HHV-6 has been found in histiocytes in some cases of RDD, but the relationship between the disorder and these viruses remains unclear.

On CT, the dominant finding is lymphadenopathy, which can involve hilar, mediastinal, intrapulmonary, and axillary nodal stations. The lungs may demonstrate cystic or interstitial 
disease. Pleural effusions and airway disease, manifested as air trapping and bronchiectasis may also be seen in RDD.

\section{Clinical history}

A 65-year-old woman, who is a former smoker, presented for evaluation of chronic cough and was found to have multiple pulmonary nodules in the right middle and lower lobes on chest $\mathrm{CT}$, largest measuring $1.4 \mathrm{~cm}$, with associated right hilar lymphadenopathy (Figs. 1 and 2). These findings were suspicious for malignancy, for which a wedge resection of the lesions, accompanied by lymph node dissection, was performed.

\section{Methods}

After excision of the lesion, histopathologic examination was performed, accompanied by pertinent special stains and immunohistochemistry.

\section{Results}

On excision, the lung parenchyma showed an extensive intraalveolar lymphohistiocytic infiltrate with occasional neutrophils, accompanied by extensive peribronchial, perivascular, and subintimal involvement. There was marked destruction of medium to large arterial vessels, with partial to complete obliteration of their lumen (Fig. 3a-f). At higher magnification, histiocytes with emperipolesis, more evident in the subintimal portion of the vessels, were noticed and highlighted by S-100

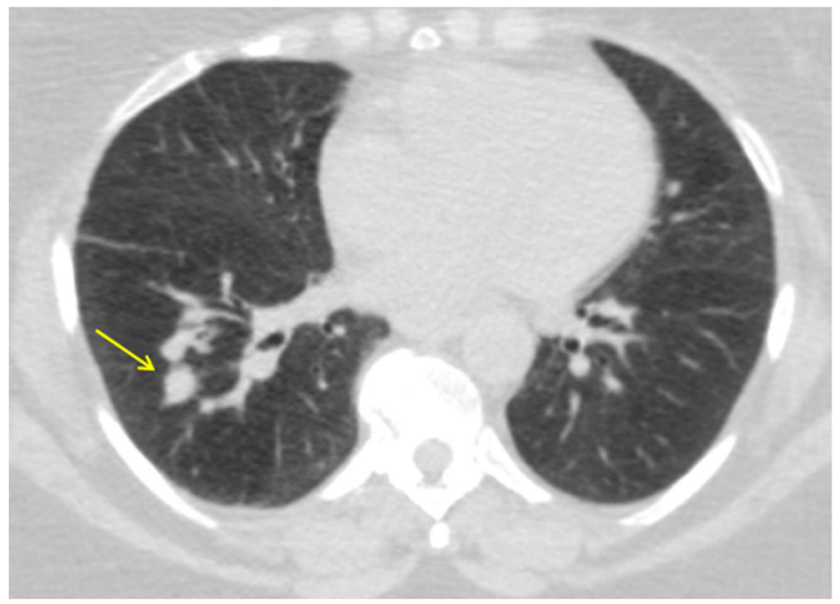

Fig. 1 CT scan of the chest in lung windows shows two, wellcircumscribed pulmonary nodules in the right lower lobe (arrow), the largest measuring $1.4 \mathrm{~cm}$ in greatest dimension

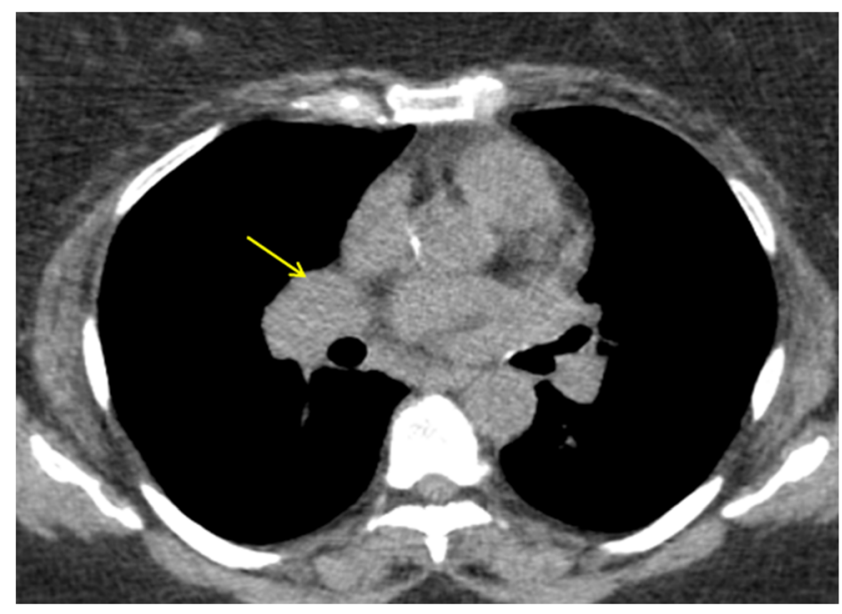

Fig. 2 CT scan of the chest in soft tissue window shows right hilar lymphadenopathy

immunohistochemical staining, while negative for CD1a (Fig. 3).

Sections of the hilar lymph nodes showed marked sinus histiocytosis with noticeable emperipolesis, consistent with nodal RDD, as well. Positive immunohistochemical staining for S-100 and negativity for CD1a confirmed these findings.

Special stains for microorganisms (Ziehl-Nielsen, Gomori Methenamine Silver) and in situ hybridization for Epstein-Barr virus-encoded RNA were negative. Immunohistochemical staining for IgG and IgG4 ruled out the presence of IgG4-related sclerosing disease. The absence of staining for Epstein-Barr virus-encoded RNA (EBER) also helped rule out lymphomatoid granulomatosis.

Six months after excision of the lesion, the patient has not shown additional symptoms or recurrence of the lung nodules on follow-up imaging.

\section{Discussion}

Parenchymal involvement of the lung by RDD is relatively rare. In a series of nine patients with intrathoracic manifestations of RDD [5], the most common histologic finding was mediastinal lymphadenopathy, followed by interstitial infiltrates, pleural effusion, and cysts. To our knowledge, vasculitis in the setting of RDD has only been reported three times in the literature, once as an intracranial lesion obstructing the carotid artery [6], and twice as lesions involving the aorta [7] and/or main pulmonary artery [7, 8]. To date, RDD involving distal pulmonary vessels has not been reported in the English literature.

Recognition of RDD is important to prevent unnecessary aggressive treatment, given the fact that these potentially lifethreatening lesions can be treated by surgical excision alone, 
Fig. 3 Histologic sections of the lung nodules show a mixed mononuclear inflammatory infiltrate comprising the perivascular and subintimal spaces (a). A Van Giesson stain highlights the elastic lamina of these arteries (b). Higher magnification shows occassional histiocytes with well-preserved small lymphocytes within their cytoplasm (emperipolesis) (c, arrow). An immunohistochemical stain for S-100 highlights the lesional histiocytes, some showing emperipolesis (arrows), in a subintimal and perivascular distribution (d, e). The same mononuclear inflammatory infiltrate is noticed in a peribronchial distribution (f). Concomitant nodal disease is noticed in the hilar lymph nodes, which show sinusal expansion with abundant histiocytes with emperipolesis (g, h)

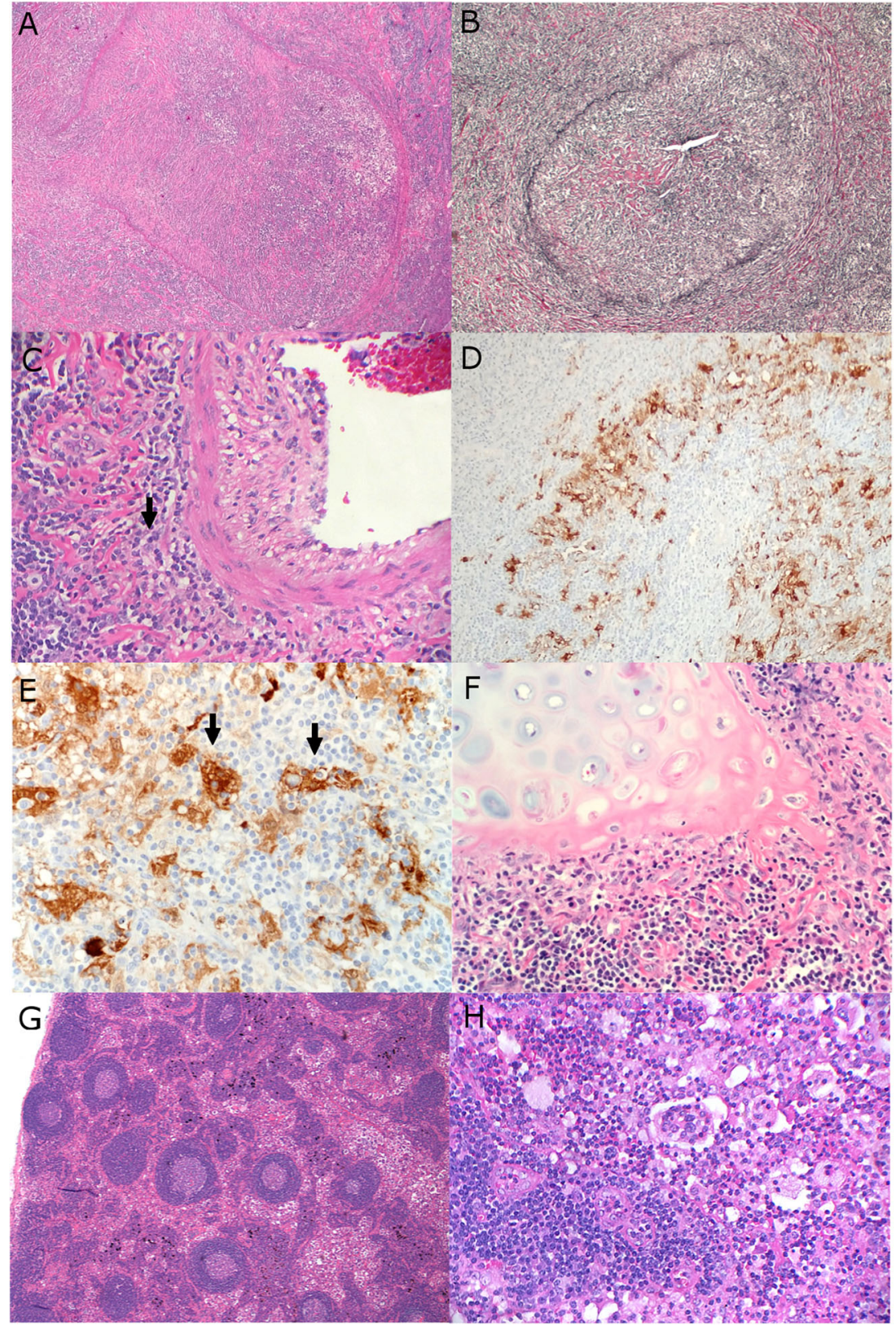

without need for radiation or chemotherapy [3, 4, 9]. In order to achieve an adequate diagnosis, clinical suspicion and careful morphologic evaluation, in addition to pertinent immunohistochemical studies, are necessary.

Other rare lymphoproliferative diseases such as lymphomatoid granulomatosis must be differentiated by performing in situ hybridization for EBER [10]. Langerhans cell histiocytosis, ANCA-associated disease (Wegener's granulomatosis), and sarcoidosis, among others, are additional differential considerations, for which additional testing must be performed [5]. There are morphologic and clinical overlapping features between RDD which include IgG4-related sclerosing disease, including the presence of storiform fibrosis and, as in this case, obliterative vasculitis; however, this relationship has not been clearly understood [11].

\section{Compliance with ethical standards}

Funding This study received no external funding.

Ethical approval This article does not contain any studies with human participants or animals performed by any of the authors.

Conflict of interest The authors declare that they have no conflicts of interest. 


\section{References}

1. Rosai J, Dorfman RF (1969) Sinus histiocytosis with massive lymphadenopathy. A newly recognized benign clinicopathological entity. Arch Pathol 87(1):63-70

2. Foucar E, Rosai J, Dorfman R (1990) Sinus histiocytosis with massive lymphadenopathy (Rosai-Dorfman disease): review of the entity. Semin Diagn Pathol 7(1):19-73

3. Mantilla JG, Goldberg-Stein S, Wang Y (2016) Extranodal RosaiDorfman disease: clinico-pathologic series of thirteen ten cases patients with radiologic correlation and review of the literature. Am J Clin Pathol 145(2):211-21

4. Gaitonde S (2007) Multifocal, extranodal sinus histiocytosis with massive lymphadenopathy: an overview. Arch Pathol Lab Med 131(7):1117-21

5. Cartin-Ceba R, Golbin JM, Yi ES, Prakash UB, Vassallo R (2010) Intrathoracic manifestations of Rosai-Dorfman disease. Respir Med 104(9):1344-9
6. Leung JLY, Cheung JYL, Tan TC, Tang KW, Chan CM, Ho LC, Chan SCH (2003) Carotid artery occlusion in a patient with intracranial Rosai-Dorfman disease. Hong Kong J Radiol 6:211-3

7. Prendes BL, Brinkman WT, Sengupta AL, Bavaria JE (2009) Atypical presentation of extranodal Rosai-Dorfman disease. Ann Thorac Surg 87(2):616-8

8. Walters DM, Dunnington GH, Dustin SM, Frierson HF, Peeler BB, Kozower BD, Ailawadi G, Jones DR, Lau CL (2010) RosaiDorfman disease presenting as a pulmonary artery mass. Ann Thorac Surg 89(1):300-2

9. Zhu F, Zhang JT, Xing XW, Wang DJ, Zhu RY, Zhang Q, Wang HT, Lang SY (2013) Rosai-Dorfman disease: a retrospective analysis of 13 cases. Am J Med Sci 345(3):200-10

10. Colby TV (2012) Current histological diagnosis of lymphomatoid granulomatosis. Mod Pathol 25(Suppl 1):S39-42

11. Kuo TT, Chen TC, Lee LY, Lu PH (2009) IgG4-positive plasma cells in cutaneous Rosai-Dorfman disease: an additional immunohistochemical feature and possible relationship to IgG4-related sclerosing disease. J Cutan Pathol 36(10):1069-73 